\title{
EL CONVENIO DE ESTAMBUL Y LOS SUJETOS DE LA VIOLENCIA DE GÉNERO. EL CUESTIONAMIENTO DE LA VIOLENCIA DOMÉSTICA COMO CATEGORÍA JURÍDICA
}


SUMARIO

1. INTRODUCCIÓN. 2. RELACIÓN ENTRE PATRIARCADO Y VIOLENCIA DE GÉNERO: EL CONCEPTO DE VIOLENCIA DE GÉNERO. 3. LOS HOMBRES, SUJETOS ACTIVOS DE LA VIOLENCIA DE GÉNERO. 4. LA CONFUSIÓN CONCEPTUAL DE LA VIOLENCIA DE GÉNERO EN EL CONVENIO DE ESTAMBUL: LA CATEGORIZACIÓN Y LOS SUJETOS DE LA VIOLENCIA DOMÉSTICA. 


\title{
EL CONVENIO DE ESTAMBUL Y LOS SUJETOS DE LA VIOLENCIA DE GÉNERO. EL CUESTIONAMIENTO DE LA VIOLENCIA DOMÉSTICA COMO CATEGORÍA JURÍDICA
}

\author{
ASUNCIÓN VENTURA FRANCH ${ }^{1}$ \\ Profesora de Derecho Constitucional \\ Universitat Jaume I
}

\section{INTRODUCCIÓN}

Los datos sobre la violencia de género son devastadores: el informe ${ }^{2}$ de la UE sobre violencia de género de 2014 señala que 13 millones de mujeres en la UE experimentaron violencia física y 3,7 millones experimentaron violencia sexual, en la pareja (actual o pasada) o fuera de ella, durante los 12 meses previos a la entrevista de la encuesta. Esa cifra equivale a un $7 \%$ y $2 \%$ respectivamente de

1 Asunción Ventura Franch. Departamento de Derecho Público. Edificio JC2. Facultad de Ciencias Jurídicas y Económicas. Universitat Jaume I. Avda. de Vicent Sos Baynat, s/n. E-12071 Castelló de la Plana. España (Spain) Email: ventura@dpu.uji.es

2 Realizado por EUROPEAN UNION AGENCY FOR FUNDAMENTAL RIGHTS (http://fra.europa.eu/ sites/default/files/fra-2014-vaw-survey-factsheet_es.pdf. Página consultada el 14/03/16). El estudio llevado a cabo por la Agencia Europea de los Derechos Fundamentales (FRA), surge de la petición de recoger datos comparables sobre violencia de género formulada por la Presidencia Española del Consejo de la UE de 2010 y reiterada por el Parlamento Europeo, según se acredita en Ministerio de Sanidad, Servicios Sociales e Igualdad, «Información de la Delegación del Gobierno para la violencia de género», Madrid, 10 de marzo de 2014, http://www.violenciagenero.msssi.gob.es/laDelegacionInforma/pdfs/F_R_A.pdf, página consultada el 17/03/16. 
mujeres con edades de entre 18-74 años en la UE. Los porcentaje ascienden al $31 \%$ si se considera la violencia física y al $11 \%$ si se considera la violencia sexual sufridas por las mujeres europeas alguna vez en sus vidas desde que tenían 15 años. Una de cada veinte mujeres europeas ( $5 \%$ ) ha sido violada alguna vez desde que tenía 15 años.

Los porcentajes tan elevados de violencia suponen un mal endémico que está inserto en la propia estructura de la sociedad, porque no es casual que todos los países europeos y también países de fuera de Europa tengan ese nivel coincidente de violencia, como se señala por ONU MUJERES ${ }^{3}$.

Además, podemos afirmar sin riesgo a equivocarnos que los porcentajes de violencia que se ejerce contra las mujeres son muy superiores a los que se reflejan en esta encuesta, lo que quedaría patente si realmente se computaran todas las violencias que sufren las mujeres en cualquier situación y ámbito. Pero para poder visibilizarlo se tendrían que establecer antes conceptos básicos, acerca de lo que es la violencia de género y sus diferentes tipologías, que permitieran una comparación de los datos.

La falta de un concepto común impide que algunas violencias contra las mujeres se computen, al no estar incluidas en los parámetros que ahora se utilizan.

La consecuencia que de ello se deriva es que la dispersión terminológica y conceptual acaba siendo uno de los motivos que dificulta la erradicación de la violencia: si el concepto de partida es erróneo, su regulación jurídica y las medidas fácticas que se adopten no van a ser efectivas.

La violencia de género es una consecuencia de la situación de subordinación que padecen las mujeres, y esta violencia puede producirse tanto en el contexto familiar como en cualquier otro tipo de relaciones fuera de la familia, sin que necesariamente medien relaciones de afectividad.

Sin embargo, el desarrollo legislativo que se ha producido en los últimos años en diferentes países, ha centrado la violencia contra las mujeres en el ámbito de las relaciones familiares y afectivas. No existe una legislación en el ámbito de la Unión Europea que defina lo que es la violencia de género, y los países que la componen tienen legislaciones muy dispares acerca del concepto de violencia de género. De los veintisiete países que forman parte de la Unión Europea sólo Alemania, Suecia y España tienen leyes cuya denominación se aparta de la violencia doméstica; el resto regula la violencia vinculada al ámbito de la familia.

${ }^{3}$ La violencia de género es un mal generalizado que acaba afectando a una de cada tres mujeres al menos una vez en la vida. Se estima que el $35 \%$ de mujeres ha sufrido violencia física o sexual (UNWOMEN, Hechos y Cifras: acabar con la violencia contra mujeres y niñas, http://www.unwomen. org/es/what-we-do/ending-violence-against-women/facts-and-figures, página consultada el 18/03/16). 
Incluso en los países en que la legislación parece más avanzada y regulan la violencia vinculada a la subordinación de las mujeres, restringen la aplicación de sus normas a las relaciones afectivas, como ocurre en caso del Estado Español ${ }^{4}$.

Pero la violencia de género no puede ser considerada como una patología social que se reduce al ámbito privado. Al contrario, constituye una manifestación de la desigualdad de mujeres y hombres existente en nuestra sociedad. En realidad se trata de una violencia basada en el hecho de ser mujer como se ha reconocido en la Recomendación General núm. 19 adoptada por el Comité de la Convención para la eliminación de toda forma de discriminación contra la mujer en 1992; en la Declaración sobre la eliminación de la violencia contra la mujer, adoptada en diciembre de 1993 por la Asamblea General de Naciones Unidas; en la Declaración de la Cuarta Conferencia Mundial sobre la Mujer celebrada en Beijing en 1995; y en la resolución del Parlamento Europeo sobre Tolerancia Cero de 1997.

Las mujeres que son víctimas de la violencia de género sufren un grave atentando contra buena parte de sus derechos fundamentales: derecho a la igualdad y a la prohibición de toda clase de discriminación, derecho a la vida y a la integridad física y moral, derecho a la libertad y a la seguridad, entre otros. Igualmente ven afectados otros derechos que conectan con el bienestar de la persona y su familia: derecho al trabajo, derecho a la protección social de la familia y de los hijos, derecho a prestaciones sociales suficientes ante situaciones de necesidad... etc. En realidad, la situación de especial vulnerabilidad que sobreviene tras un episodio de violencia de género afecta tanto al ámbito personal, como al familiar, al económico, al laboral, al sanitario y al asistencial.

Estos derechos cuentan con reconocimiento tanto en el ámbito internacional $(\mathrm{ONU}, \mathrm{UE}, . .$.$) como en el constitucional de la mayoría de los Estados democrá-$ ticos; sin embargo, su vulneración, de forma sistemática y de manera tan extensa, no ha sido considerada, hasta el momento, motivo suficiente para la adopción de medidas más drásticas, que puedan contribuir de una manera eficiente a restablecer los derechos vulnerados.

Es imprescindible que se garantice a las mujeres el derecho a una vida libre de violencia en cualquiera de los ámbitos dónde desarrollan sus actividades per-

${ }^{4}$ La Ley Orgánica 1/2004, de 28 de diciembre, de Medidas de Protección Integral contra la Violencia de Género, en su artículo 1.1, establece que «La presente Ley tiene por objeto actuar contra la violencia que, como manifestación de la discriminación, la situación de desigualdad y las relaciones de poder de los hombres sobre las mujeres, se ejerce sobre éstas por parte de quienes sean o hayan sido sus cónyuges o de quienes estén o hayan estado ligados a ellas por relaciones similares de afectividad, aun sin convivencia». Esta fórmula ha sido recogida en muchas de las leyes de las CCAA sobre violencia de género. 
sonales, laborales, políticas e institucionales. Esto no será posible si no se aborda un concepto de violencia que incluya a cada uno de ellos.

Para establecer un concepto de violencia que pueda contribuir a avanzar en la eliminación de esa lacra social que mina los propios fundamentos del estado, al no poder garantizar los derechos fundamentales de una gran parte de la ciudadanía, se necesita analizar el origen de la violencia de género, los sujetos activos y pasivo de la misma y las diferentes manifestaciones de la violencia. Y en función de estos presupuestos se deben adoptar medidas fácticas y legales.

Recientemente ha entrado en vigor el Convenio del Consejo de Europa sobre prevención y lucha contra la violencia contra la mujer y la violencia doméstica hecho en Estambul el 11 de mayo de 2011 (BOE de 6 de junio de 2014), también conocido como Convenio de Estambul. Desde el punto de vista de las garantías representa un nivel de protección muy alto en el ámbito internacional; se trata de un instrumento cuya finalidad es contribuir a avanzar en la erradicación de la violencia de género en los países europeos ${ }^{5}$. Sin embargo, el análisis del concepto de violencia incluido en el mismo puede plantear algunos problemas, de interpretación y aplicación, a la vez que devenir ineficaz en relación al objetivo de crear una Europa libre de violencia contra la mujer.

Las normas pueden contribuir de manera importante a erradicar la violencia contra las mujeres, pero una cuestión que va a ser determinante para su efectividad es conocer y ahondar en los conceptos de género, violencia y patriarcado. Evidentemente, resulta imprescindible para poder entender las causas que motivan la condición de sujeto activo y pasivo en los casos de violencia de género así como sus consecuencias sociales y jurídicas.

\section{RELACIÓN ENTRE PATRIARCADO Y VIOLENCIA DE GÉNERO: EL CONCEPTO DE VIOLENCIA DE GÉNERO}

La violencia de género es un término muy usado para designar algunas de las agresiones que los hombres infringen a las mujeres, pero no tiene un significado unánime; en la mayoría de los casos se utiliza de forma incorrecta, identificando violencia de género con algunos de $\operatorname{los}_{\text {tipos }}{ }^{6}$ en los que se manifiesta.

5 Lousada Arochena, J. F. (2014). «El Convenio del Consejo de Europa sobre prevención y lucha contra la violencia contra las mujeres y la violencia de género». AequAlitas. Revista Jurídica de Igualdad de Oportunidades de Mujeres y Hombres, núm. 35, pp. 6-15.

${ }^{6}$ La declaración sobre la eliminación de la violencia contra la mujer. Resolución de la Asamblea General 48/104, ONU, 1994. Define la violencia contra la mujer como «todo acto de violencia basado en el género que tiene como resultado posible o real un daño físico, sexual, psicológico, 
Podría considerarse falto de interés intentar acotar un concepto a través del cual se puedan reconducir todas estas agresiones desde el punto de vista conceptual, si no tuviéramos en cuenta las consecuencias tan nefastas que ello puede tener para la erradicación de la violencia de género. Como muy bien señala Celia Amorós ${ }^{7}$ es necesario conceptualizar para dar nombre a unos determinados grupos de fenómenos y pasar a categoría lo que antes eran simples anécdotas. En definitiva, los conceptos resultan de una gran utilidad para designar una realidad y a la vez poder comprenderla.

La violencia contra las mujeres es mucho mayor en número de víctimas de lo que en realidad señalan las estadísticas, porque al no existir un concepto unificador de la violencia de género, los datos se fragmentan y parece que su alcance sea menor. Aun así, nos parecen escalofriantes los que reflejan las mujeres muertas por su pareja o expareja, en España o en cualquier otro país; pero si a esto se añade la violencia que las mujeres sufren en el trabajo, en la política, en la economía y en cualquier tipo de relación, quizá el resultado podría ser insostenible desde cualquier tipo de análisis y cuestionar los propios fundamentos del Estado.

Para comprender y conceptualizar la violencia contra las mujeres es necesario acudir al concepto de patriarcado, en cuya estructura reside el origen de esta violencia. Para ello, adoptaremos dos definiciones: una de carácter general, que afecta a la totalidad de un sistema; y otra más concreta, que detalla algunas situaciones que en principio parecen contradictorias con el sistema patriarcal pero que sin embargo son consecuencia del mismo.

incluidas las amenazas, la coerción o la privación arbitraria de la libertad, ya sea que ocurra en la vida pública o en la vida privada». Esta violencia incluye la violencia física, psicológica y sexual. Esta tipología ha sido ampliada a propuesta del grupo de especialistas para combatir la violencia de las mujeres en el Consejo de Europa (1997) añadiendo además de las ya señaladas la violencia económica, violencia estructural, violencia espiritual, violencia política e institucional y violencia simbólica. Para ampliar esta temática se puede consultar el trabajo realizado por la doctora Ferrer, V. (2007). «Las diversas manifestaciones de la violencia de género», en Bosch, E. (comp.), La violencia de género: algunas cuestiones básicas, Jaén, Editorial Formación Alcalá, pp. 61-106.

7 Amorós, C. (2008). «Conceptualizar es politizar», en Laurenzo Copello, P.; Maqueda Abreu, M.L, Rubio Castro, A. (coords.), Género, violencia y derecho, Valencia, Tirant lo Blanch, pp. 16-25. La autora apoyándose en Kant plantea que los conceptos sin datos empíricos que los avalen son vacíos, pero que los datos empíricos sin conceptos son ciegos, señala que la resignificación del lenguaje ha sido una de las armas de los oprimidos muy utilizada por el movimiento feminista propone hablar de terrorismo patriarcal, terrorismo sexista y de terrorismos de género. p. 17. 
La primera definición de carácter general corresponde a Celia Amorós ${ }^{8}$, que entiende por patriarcado un modo de dominación de los varones sobre las mujeres que tiene efectos sistémicos. La segunda, de carácter más concreto pero que tiene un gran interés para comprender las formas y los mecanismos que adopta ese modo de dominación es de Alda Facio", quien define el patriarcado «como el poder de los padres; un sistema familiar, social, ideológico y político mediante el cual los hombres, por la fuerza, usando la presión directa o por medio de símbolos, ritos tradicionales, leyes, educación, el imaginario popular o inconsciente colectivo, la maternidad forzada, la heterosexualidad obligatoria, la división sexual del trabajo y la historia robada, determinan qué funciones podemos o no desempeñar las mujeres. En este sistema, el grupo, casta o clase compuesto por mujeres, siempre está subordinado al grupo, casta o clase compuesto por hombres, aunque pueda ser que una o varias mujeres tengan poder, hasta mucho poder como las reinas o las primeras ministras, o que todas las mujeres ejerzan cierto tipo de poder, como es el poder que ejercen las madres sobre los y las hijas».

La concreción de esta definición de patriarcado permite, por una parte, visualizar que este poder puede utilizar todas las formas de sometimiento, incluida la violencia, y que además no excluye la posibilidad de que algunas mujeres participen individualmente del poder patriarcal, o que tengan un cierto tipo de poder que el patriarcado no considera como tal.

El concepto de patriarcado ha sido «resignificado» por la teoría feminista y además ha sido desnaturalizado, contribuyendo con ello, en gran medida, al cuestionamiento del patriarcado como sistema de dominación incompatible con los sistemas democráticos. Una de las razones que había contribuido a la consolidación y permanencia del patriarcado ha sido la teorización y justificación de que formaba parte de la naturaleza humana, lo que contribuía a su aceptación como algo irremediable y la vez inmodificable. La teoría feminista aporta elementos científicos suficientes para entender que el que haya existido de forma generalizada no quiere decir que no pueda dejar de existir ${ }^{10}$.

8 Amorós, C. (2008). «Conceptualizar es politizar», op. cit., p. 18.

9 FACIO, A. (1993). «El derecho como producto del patriarcado», en FACIO, A. y CAMACHO, R. (editoras) Sobre patriarcas, jerarcas, patrones y otros varones (Una mirada de género sensitiva del derecho), San José de Costa Rica, Ilanud: Programa Mujer, Justicia y Género, pp. 7-29.

${ }^{10}$ BARRÈre, M. A. (2008), «Género, discriminación y violencia contra las mujeres», en Género, violencia y derecho, op. cit., pp. 27-47. 
$\mathrm{Al}$ concepto de género se le atribuyen orígenes diferentes ${ }^{11} \mathrm{y}$, como señala Rosa $\mathrm{Cobo}^{12}$, se ha desarrollado en varias direcciones, pero señalaremos dos relacionadas con este trabajo. En primer lugar, «género» se refiere a la existencia de una normatividad femenina edificada sobre el sexo como hecho anatómico. Y en segundo lugar, esta normatividad femenina reposa sobre un sistema social en el que el género es un principio de jerarquización que asigna espacios y distribuye recursos a varones y mujeres. Este sistema social será designado por la teoría feminista con el término patriarcado.

Gayle Rubin ${ }^{13}$ define el sistema sexo/género como un conjunto de disposiciones por el que una sociedad transforma la sexualidad biológica en productos humanos. Así el sexo lleva la marca de la biología y el género la marca de la cultura. «Es el conjunto de prácticas, símbolos, representaciones, normas y valores sociales que las sociedades elaboran a partir de la diferencia sexual». La estructuración del sistema sexo/género comporta necesariamente la represión de aquellas conductas que se aparten del modelo. La violencia de género presupone una posición de poder de los hombres en la sociedad, dado que en la estructura del sistema sexo/género, el sexo que ejerce el poder es el masculino, y fruto de esa posición se ejerce la violencia contra las mujeres con el ánimo de control y subordinación.

La cultura ha establecido un sistema de organización sobre una base biológica por la que las mujeres, históricamente, han sido dominadas y se han encontrado en una posición de subordinación y los hombres en una posición de dominación. Para analizar este situación es necesario partir de la existencia de estas dos posiciones claramente diferenciadas pero, a la vez, estrechamente vinculadas, hasta el punto que no se puede entender la una sin la otra: para que un grupo esté subordinado debe existir otro grupo dominante. La subordinación de las mujeres —al igual que cualquier otro tipo de subordinación — se produce porque otros, apoyados por todos los instrumentos del poder, ejercen la función de dominio. Por ello, es necesario analizar las relaciones que se dan entre la posición

${ }^{11}$ Izquierdo, M. J. (1998). El malestar de la desigualdad, Madrid, Cátedra-feminismos. La autora atribuye el origen del concepto de género a la psiquiatría. Pero señala la posición de otras autoras, como Silvia Turbet, que lo atribuye a la endocrinología infantil. Sin embargo reconoce que existe un amplio consenso en identificar el concepto de género con las teorías de la antropóloga feminista Gayle Rubin (1975), que a partir de ese momento se convierte en una categoría central del pensamiento feminista (p. 53).

${ }^{12}$ Сово, R. (2008). «El género en las ciencias sociales», en Género, violencia y derecho, op. cit., p. 53.

13 Rubin, G.; Glover, D.; Kaplan, C. (2002). Géneres i identitats culturals, Vic, Editorial Eumo, p. 34. 
subordinada y la posición de dominante, y las consecuencias que han tenido y la dificultad que comporta intentar, a partir de esta estructura, poder establecer unas relaciones igualitarias. Simplificando, podríamos señalar que hay dos grandes bloques de funciones en la historia de la humanidad, en la que los hombres, por tener un sexo determinado, han realizado las que tienen más valor, las del dominante, mientras que las mujeres, por tener un sexo diferente al del hombre, han realizado todas aquellas que socialmente tienen un reconocimiento menor. Las diferentes asignaciones de funciones sociales en base al sexo han generado una estructura social marcada por la subordinación y la discriminación de las mujeres en todos los ámbitos.

Por tanto, el género ${ }^{14}$ no puede identificarse con mujer ni tampoco con sexo, aunque el hecho de tener un sexo femenino es la base de la asignación de funciones que tienen una peor valoración. El género no puede confundirse con el hecho de ser mujer porque en primer lugar hay dos géneros, masculino y femenino con posiciones diferentes como hemos visto; y en segundo lugar, porque un género no puede explicarse solo, dado que necesita del otro para su existencia ${ }^{15}$. La asignación de funciones diferentes en base al sexo y la jerarquización de los géneros es una cuestión que se nutre de razones culturales e históricas y sobre la que se ha sustentado la construcción social y todavía condiciona el modo de percibir la realidad.

La modernidad ha introducido valores y principios que, «reconceptualizados» desde la teoría feminista, cuestionan la jerarquización de los géneros masculino y femenino; pero, a pesar de los avances producidos en los últimos años, todavía no se ha construido un modelo alternativo al sistema sexo/género capaz de eliminar la dominación versus subordinación, y establecer un sistema cuyo fundamento sea la persona y no su sexo.

${ }^{14}$ Sobre las distintas acepciones de la categoría género se puede consultar la obra de BoDELÓN, E. (2012). Violencia de género y las respuestas de los sistemas penales, Barcelona, Ediciones Didot, pp. 14-26.

15 Turbet, S. (2008). «La crisis del concepto de género», en Género, Violencia y Derecho, op cit., p. 99. En los últimos tiempos se utiliza mucho la palabra género porque parece que sea un término más fácilmente aceptado y así se evita hablar de patriarcado, también y de alguna manera, se pretende invisibilizar al feminismo como teoría critica que reconceptualizó el patriarcado, e incluso, el termino género. Además hay una creencia, por cierto cada día más extendida, que identifica género con mujeres y así nos podemos encontrar con algunos estudios que se denominan de género y en realidad solamente hablan de mujeres, y no establecen una relación dialéctica entre ambos, resultando que son estudios parciales y no pueden contribuir a la eliminación de la discriminación de las mujeres. La utilización de género se cuestiona tanto desde el feminismo de la diferencia como desde el feminismo de la igualdad porque representa una abstracción voluntarista en detrimento de la representación sexual y del conflicto inherente a ella. 
Los valores y principios que sirvieron de base para establecer un nuevo modelo de sociedad, sobre todo el principio de igualdad, no estaban pensados para construir un nuevo modelo de relaciones sino para ordenar un sistema político en el que los hombres fueran libres en el ámbito privado e iguales en el ámbito público. La exclusión de las mujeres en un primer momento formaba parte de la «naturalización» ${ }^{16}$ del proceso, y así se interiorizó en la idea de igualdad, sobre la base de un modelo exclusivamente masculino.

La exclusión de las mujeres del ámbito público fue cuestionada por John Stuart Mill y Harriet Taylor ${ }^{17}$, quienes, siguiendo la teoría de autoras anteriores (concretamente a François Poullain de la Barre ${ }^{18}$ y Mary Wollstonecraft ${ }^{19}$ ) argumentan la necesidad de que las mujeres y los hombres sean iguales, y señalan la exclusión de aquellas como un prejuicio debido a las costumbres. Una aportación significativa de Mill y Tailor es que el sexo y el ámbito emocional hacen que la dominación de los hombres sobre las mujeres sea una dominación muy diferente a las demás. Los hombres no sólo quieren la obediencia de las mujeres sino también sus sentimientos y si no lo consiguen utilizan la violencia contra ellas ${ }^{20}$.

La violencia ha sido una forma de resolución de conflictos sobre todo en las sociedades premodernas, pero la llegada de las sociedades modernas hace que se vaya deslegitimando y que se articulen formas diferentes de cohesión social. Sin embargo, los pensadores modernos (Look, Rousseau, Kant...), al teorizar sobre la inferioridad de las mujeres, les aplican los mismos argumentos en los que se sostenía la existencia de una sociedad estamental en la premodernidad. Ello

16 Amorós, C. (2008). «Conceptualizar es politizar», op. cit., p. 20. La autora señala que el movimiento feminista se ha encargado de desnaturalizar, cuestionándola, la configuración histórica de la dicotomía público-privado.

17 Mill, J. S.; Taylor Mill, H. (2001). Ensayos sobre la igualdad Sexual, intr. de Campillo, N., Madrid, Cátedra; Campillo, N. (I986). «J. St. Mill: Igualdad, criterio de la modernidad», en Durán Heras, M. Á. (coord.), Mujeres y hombres en la formación de la teoría sociológica, Madrid, Centro de Investigaciones Sociológicas, pp. 73-112.

18 Poullain de la Barre, F. (1993). De la educación de las damas: para la formación del espiritu en las ciencias y en las costumbres, Madrid, Cátedra; De l'Égalité des deux sexes, discours physique et moral où l'on voit l'importance de se défaire des préjugés, Paris, Chez Jean du Puis, 1673, rééd. Fayard, 1984: Gallimard, 2015.

19 Wollstonecraft, M. (1977). Vindicación de los derechos de la mujer, Madrid, Debate; Ventura Franch, A. (1993). «Mary Wollstonecraft: una aproximación a su obra». Asparkia: investigación feminista, núm. 2, http://www.raco.cat/index.php/Asparkia/article/view/107293 (página consultada el 18/09/16).

${ }_{20}$ Mill, J. S. (1997), The subjection of woman, Bristol, Tommes. La relación de la obra de John Stuart Mill con la violencia de género es analizada por la profesora Juana María Gil Ruiz en el artículo «John Stuart Mill y la Violencia de Género: las trampas de la educación diferencial». Anuario de filosofía del derecho, núm. 23, 2006, pp. 61-84. 
comporta que indirecta y/o directamente se mantuviera para ellas esa forma de la resolución de los conflictos ${ }^{21}$ y supone que la violencia contra las mujeres entra como referente normativo en el discurso de la modernidad ${ }^{22}$.

Las mujeres son excluidas del proyecto de la modernidad y recluídas al ámbito privado; los grandes principios de este nuevo orden aparentemente involucran a todas las personas, incluidas en un sujeto universal ${ }^{23}$ que en realidad se identifica con los hombres, o mejor dicho, con algunos hombres que reúnan las características de ser blancos y propietarios ${ }^{24}$. La ampliación formal del sujeto universal a través de la inclusión de otros hombres, en primer lugar los blancos y no propietarios, más tarde los hombres negros y, finalmente, a las mujeres, es cuestionada desde una perspectiva de la igualdad material, dado que éstas no tienen condiciones de igualdad y sobre ellas pesa la autoridad de los hombres y la obediencia o el castigo.

La violencia que los hombres ejercen sobre las mujeres resultó ser un motivo de gran preocupación para el movimiento feminista. Desde los años sesenta del siglo pasado, este movimiento dirige uno de sus focos de atención a la violencia sexual y a la violencia contra las mujeres, en un doble sentido: por una parte,

21 De Miguel, A. (2015). Neoliberalismo sexual. El mito de la libre elección, Madrid, Ediciones Cátedra, Feminismos, pp. 252-254.

22 PosadA, L. (2001). «Las hijas deben ser siempre sumisas (Rousseau). Discurso patriarcal y violencia contra las mujeres», en BERNÁrDEZ, A. (ed.), Violencia de género y sociedad: una cuestión de poder, Ayuntamiento de Madrid.

23 Sobre el cuestionamiento del sujeto universal como omnicomprensivo de hombres y mujeres existe una abundante literatura desde el punto de vista filosófico, siendo la más relevante la de las obras de Celia Amoros Puente. Caben citar, entre otras, (1997). Tiempo de feminismo: sobre feminismo, proyecto ilustrado y postmodernidad (i.e. Postmodernidad), Madrid, Cátedra; (1995). Hacia una crítica de la razón patriarcal, Círculo de Lectores; (2005). La gran diferencia y sus pequeñas consecuencias - para las luchas de las mujeres, Madrid, Cátedra. También a Pateman, C. (1995) El contrato sexual. Barcelona. Antropos.

Desde el punto de vista jurídico-político se pueden consultar las obras de las profesoras MARÍA Luisa Balaguer Callejón [(2005). Mujer y constitución: la construcción jurídica del género, Madrid, Cátedra; y (2010). Igualdad y Constitución Española, Madrid, Tecnos]; Julia Sevilla Merino [(2004). Mujeres y ciudadanía: la democracia paritaria, València, Institut Universitari d'Estudis de la Dona] y Mar Esquembre Valdés [(2010). «Género, ciudadanía y derechos. La subjetividad política y jurídica de las mujeres como clave para la igualdad efectiva. Corts: Anuario de Derecho Parlamentario, núm. 23, pp. 47-85].

24 Ventura Franch, A. (1999). Las mujeres y la Constitución Española de 1978, Madrid, Instituto de la Mujer; Sevilla Merino, J. y Ventura Franch, A. (I998). «El sexo del Estado», en Portal Nieto, A. M. (ed.), Mujeres, derecho, participación política y empleo, pp. 153-171; Sevilla Merino, J., Ventura Franch, A., y García Campé, S. (i997). «La igualdad efectiva entre mujeres y hombres desde la teoría constitucional». Revista del Ministerio de Trabajo e Inmigración, núm 67, pp. 63-82. 
deslegitimándola como instrumento de resolución de conflictos; y por otra, elaborando nuevas propuestas de análisis. En la década de los setenta, la violencia contra las mujeres en la pareja comenzó a denunciarse de forma específica, como problema y surgió en Inglaterra el movimiento de mujeres maltratadas, con la apertura de una casa de acogida. Poco a poco, esta práctica se fue extendiendo a otros a países europeos y en la década de los ochenta las feministas europeas comenzaron a presionar a sus respectivos gobiernos con el fin de que adoptaran medidas para la erradicación de la violencia y la protección de las mujeres maltratadas.

A partir de estos acontecimientos se va ampliando el debate y los organismos internacionales asumen la problemática, lo que conduce a la elaboración y aprobación de Declaraciones contra estas violencias ${ }^{25}$. Una de las pioneras es la Declaración de Naciones Unidas sobre la eliminación de la Violencia contra la Mujer, aprobada en diciembre de 1993 y promulgada en 1994. Esta Declaración define la violencia como una consecuencia de la situación de subordinación que padecen las mujeres. Sin embargo, además de señalar el origen de la violencia es necesario situarla en el contexto político y social actual, y analizar qué consecuencias tiene para las mujeres que la sufren, sobre todo en relación con los derechos humanos ${ }^{26}$.

Efectivamente, la violencia contra las mujeres representa una violación de los derechos humanos y existen diferentes tipos: física, psicológica, sexual... y pueden incluirse también las propias amenazas de sufrir violencia. Además, pueden producirse tanto en el contexto familiar como en cualquier otro tipo de relaciones fuera de la familia, sin que necesariamente medien relaciones de afectividad. En realidad se trata de una violencia basada en el hecho de ser mujer ${ }^{27}$.

Las Declaraciones internacionales de violencia contra las mujeres se dan tanto en el ámbito de Naciones Unidas cómo en América y Europa. Es en éste último ámbito en el que cabe destacar el Convenio del Consejo de Europa sobre

25 La primera referencia es la de la Convención de Naciones Unidas de 1979 como una forma de discriminación (http://www.un.org/womenwatch/daw/cedaw/text/sconvention.htm). También pueden citarse la Conferencia mundial sobre derechos humanos celebrada en Viena en 1993 (http:// www.un.org/es/development/devagenda/humanrights.shtml) y la Declaración de Naciones Unidas sobre la Eliminación de la violencia contra la mujer del mismo año (http://www.ohchr.org/SP/ ProfessionalInterest/Pages/ViolenceAgainstWomen.aspx). Páginas consultadas el 21/03/16.

${ }^{26}$ Figueruelo Burrieza, A. (2015). «La violencia de género: una cuestión de derechos humanos», en Gallardo Rodríguez, A. (coord.); Del Pozo Pérez, M. (dir.), ¿Podemos erradicar la violencia de género?: análisis, debate y propuestas, pp. 57-68.

27 Bosch, E.; Ferrer, V.; Ferreiro, A. y Navarro, C. (2013). La violencia contra las mujeres. El amor como coartada, Barcelona, Antropos. Las autoras mantienen a lo largo del texto que la violencia de género es una violencia contra las mujeres, por la posición de subordinación que ocupan en la estructura patriarcal. 
prevención y lucha contra la violencia contra la mujer y la violencia doméstica, de 11 de mayo de 2011, también conocido por Convenio de Estambul, ratificado por España el 18 de marzo de 2014 y en vigor desde el 1 de agosto del mismo año. Es el primer documento vinculante, en el ámbito europeo, sobre la violencia contra la mujer que introduce mecanismos para garantizar su cumplimiento.

Sin embargo, este Convenio en un intento, por otra parte loable, de acotar unos conceptos básicos acerca de la violencia contra las mujeres, elaborados fundamentalmente por la teoría feminista, los asume sólo parcialmente; y al no hacerlo con todas las consecuencias introduce algunos elementos de confusión, concentrados en su artículo 3. Tal y como desarrollaremos en el apartado siguiente, la conceptualización de la violencia contra las mujeres en el Convenio de Estambul parece huir de la tríada sobre la que se sustenta la violencia contra las mujeres, esto es, patriarcado, género y violencia.

Analizados los conceptos de patriarcado, género y violencia, entendemos que tienen una clara relación; y que cualquiera de las definiciones y conceptos sobre la violencia de género debe relacionarse con el patriarcado y con la subordinación de las mujeres. Por ello, seguiremos la definición de las profesoras Esperanza Bosch y Victoria Ferrer, y hablaremos de violencia de género o violencia contra las mujeres para hacer referencia a aquellas formas de violencia que son ejercidas por varones contra mujeres por el hecho de serlo y por la posición social que ocupan en función de su condición de mujeres en la sociedad patriarcal ${ }^{28}$.

La violencia generada por el sistema sexo/género ha sido denominada de diferentes formas. Las denominaciones de los problemas se relacionan con el grado de asunción que una sociedad determinada tiene sobre el hecho en concreto. En el caso de la violencia contra las mujeres (al igual que en otros temas, como los de la representación política, la conciliación entre la vida laboral y familiar,... etc.) la tendencia habitual es que en un primer momento se tienda a buscar denominaciones genéricas, en las que resulta difícil identificar a los sujetos que intervienen en la relación, como en el caso que nos ocupa: el agresor y la víctima. Y ello no es casual, porque de esta manera se consigue que pueda parecer que los

${ }^{28}$ No entraremos en detallar las clases de violencia porque no es objeto de este trabajo pero, no obstante, resulta de un gran interés ver sus diferentes clasificaciones. A los efectos de este trabajo seguiremos el criterio de adoptado por Bosch, E., Ferrer, V. y Almazora, A., op. cit., p. 87, de carácter amplio, que señala dos grandes ámbitos de la violencia: el que surge de las relaciones afectivas y el que surgen de las relaciones que se producen en el ámbito público, sean o no relaciones afectivas. También resulta interesante el estudio de RAQuel OsBorne (2008). «El poder del amor (o las formas sutiles de la dominación patriarcal)», en Género, violencia y derecho, op. cit., pp. 179-194, acerca de las diferentes formas de violencia que se dan en las sociedades democráticas para mantener el poder patriarcal. 
sujetos pueden ser intercambiables ${ }^{29}$. Así ocurre cuando en un primer momento la violencia contra las mujeres se denomina violencia doméstica ${ }^{30}$ : ésta denominación permite difuminar el origen de la violencia y la posición de los sujetos. Pero en la medida en que los estudios van confirmando que la violencia doméstica se fundamenta en el sistema sexo/género se dan los primeros pasos para que las organizaciones internacionales y las normas que de ellas fluyen comiencen a reconocer, y consolidar, que la violencia doméstica es un tipo concreto de violencia que tiene su raíz en las relaciones de poder establecidas en la sociedad y en las que las mujeres ocupan una posición de inferioridad respecto de los hombres. No obstante resulta complicado resolver el problema conceptual, porque es difícil establecer consensos en relación a la asunción por parte de la sociedad de que el sistema sexo/género ha consolidado un tipo de organización social que impide la implantación de una sociedad estructurada sobre la base de la igualdad de la ciudadanía, a pesar de los esfuerzos políticos y legislativos que se están realizando.

En el ámbito internacional el concepto de violencia de género que más se identifica con la propuesta realizada en este trabajo es el que define la Declaración sobre la eliminación de la violencia contra la mujer, adoptada en diciembre de 1993 por la Asamblea General de Naciones Unidas. En ella se determina el origen de la violencia en los siguiente términos: «la violencia contra la mujer constituye una manifestación de relaciones de poder históricamente desiguales entre el hombre y la mujer, que han conducido a la dominación de la mujer y a la discriminación en su contra por parte del hombre e impedido el adelanto pleno de la mujer, y que la violencia contra la mujer es uno de los mecanismos sociales fundamentales por los que se fuerza a la mujer a una situación de subordinación respecto del hombre» (apartado 6). En base a ello, el artículo 1 de la mencionada Declaración define como violencia contra la mujer «todo acto de violencia basado en la pertenencia al sexo femenino que tenga o pueda tener

29 La Magistrada que plantea la Cuestión de inconstitucionalidad sobre el art. 153.1 del CP modificado de acuerdo con la redacción dada por el art. 37 de la LOIMVG 1/2004, de 28 de diciembre, admite la posibilidad de una autoría femenina del delito de lesiones en una relación de afectividad contemplado en dicho art. 153.1 del CP., tal y como se señala en la STC 59/2008, de 14 de mayo (Fj.4).

30 Se puede señalar que la primera expresión que se utiliza para designar este tipo de violencia es el término «malos tratos»; así figura en los primeros datos estadísticos del Ministerio del Interior sobre la violencia en el ámbito familiar en 1984. Es a partir de finales de la década de los 90 que se comienza a generalizar el término violencia doméstica. El Observatorio contra la Violencia doméstica y de género del Consejo General del poder Judicial se crea en el año 2002. Con la Ley 27/2003, de 31 de julio, reguladora de la orden de protección de las víctimas de violencia doméstica, es la primera vez que se incorpora este término a una ley procesal. 
como resultado un daño o sufrimiento físico, sexual o psicológico para la mujer, así como las amenazas de tales actos, la coacción o la privación arbitraria de la libertad, tanto si se producen en la vida pública como en la privada».

El art. 2 de esta Declaración establece una tipología ${ }^{31}$ de la violencia que puede perpetrarse tanto en el ámbito público como en el privado, dejando la puerta abierta a su ampliación. Este artículo señala la violencia en el ámbito familiar o en las relaciones de afectividad como uno de los tipos de violencia contra la mujer. Así, la violencia doméstica participa del origen y de la definición de violencia contra las mujeres en la que, claramente, el sujeto que la ejerce es un hombre y mujeres las que la sufren, dado que es un acto de violencia realizado desde una posición de poder que otorga a los hombres la estructura patriarcal.

En el mismo sentido se ha pronunciado el Parlamento Europeo en su Resolución sobre Tolerancia Cero de 1997: «Considerando que la violencia contra las mujeres está sin duda alguna vinculada al desequilibrio en las relaciones de poder entre los sexos en los ámbitos social, económico, religioso y político, pese a las legislaciones nacionales e internacionales a favor de la igualdad». Sin embargo, todavía no existe un concepto unívoco de la violencia de género en los documentos legales de la Unión Europea ${ }^{32}$.

\section{LOS HOMBRES, SUJETOS ACTIVOS DE LA VIOLENCIA DE GÉNERO}

La concreción del sujeto activo y pasivo de la violencia de género ha generado y sigue generando una abundante literatura, sobre todo en el ámbito del derecho.

31 Artículo 2: Se entenderá que la violencia contra la mujer abarca los siguientes actos, aunque sin limitarse a ellos: a) La violencia física, sexual y sicológica que se produzca en la familia, incluidos los malos tratos, el abuso sexual de las niñas en el hogar, la violencia relacionada con la dote, la violación por el marido, la mutilación genital femenina y otras prácticas tradicionales nocivas para la mujer, los actos de violencia perpetrados por otros miembros de la familia y la violencia relacionada con la explotación; b) La violencia física, sexual y sicológica perpetrada dentro de la comunidad en general, inclusive la violación, el abuso sexual, el acoso y la intimidación sexuales en el trabajo, en instituciones educacionales y en otros lugares, la trata de mujeres y la prostitución forzada; 3 c) La violencia física, sexual y sicológica perpetrada o tolerada por el Estado, dondequiera que ocurra.

32 Freixes, T. y Román, L. (2014). Protección de las víctimas de violencia de género en la Unión Europea, Tarragona, Universidad Rovira i Virgili, p. 15. Las autoras demuestran en este estudio la disparidad de conceptos de la violencia de género en el ámbito de la Unión Europea. 
Los sujetos de las normas jurídicas parecen intercambiables y, en un principio, todos y todas pueden ser sujetos de las normas, de acuerdo con los principios del estado liberal en el que las normas tienen una vocación universal.

Sin embargo, este principio ya ha sido matizado por la teoría y realidad del estado social y las normas no tienen ese pretendido carácter universal; son más bien normas sectoriales, y el sujeto «todos» se refiere a todos los que tienen las características incluidas en las normas. El ejemplo más claro es el del derecho al trabajo.

El análisis y la relación entre los conceptos de violencia, género y patriarcado han permitido la identificación de los sujetos activos y pasivos de la violencia contra las mujeres. No obstante, y a pesar de los estudios y las declaraciones internacionales sobre la materia, la cuestión de si las mujeres pueden ser sujetos activos de la violencia de género no está resuelta y prueba de ello es que el debate suele reaparecer periódicamente, tal como ha ocurrido con la regulación que se establece en el Convenio de Estambul.

También en el ámbito del Derecho español esta discusión está muy presente y ha dado lugar a diversos conflictos interpretativos. Así, uno de los aspectos más controvertidos de la Ley Orgánica Integral contra la Violencia de Género se ha centrado en la regulación del Título IV, en la que se establecía una penalización mayor $^{33}$ de ciertas conductas delictivas cuyo fundamento último hay que buscarlo en el sistema sexo/género.

El Consejo General del Poder Judicial ${ }^{34}$, en su informe sobre el Anteproyecto de ley, estimaba que la agravación de las penas propuestas representaba una

33 Ver artículo 37, «Protección contra los malos tratos», de la Ley Orgánica de Medidas de Protección Integral contra la Violencia de Género 1/ 2004, de 28 de diciembre. AlguAcil GónZalez-Aurioles, J. (2005). «Tutela Penal», en Aranda, E. (dir.), Estudios sobre la ley integral contra la violencia de género, Madrid, Dykinson, pp. 113-132. El autor analiza las intervenciones realizadas por algunos expertos en el Congreso de los Diputados, en la tramitación parlamentaria de la Ley, con respecto a la tutela penal; algunos muestran su oposición, y los que la defienden la justifican como una medida de acción positiva en el ámbito del derecho penal.

34 Arroyo Zapatero, L. (2008). «La violencia de género en la pareja en el Derecho Penal Español», en Muñoz Conde, F. (edit.), Problemas actuales del Derecho penal y de la Criminología. Estudios penales en memoria de la Profesora Dra. María del Mar Díaz Pita, Tirant lo Blanch, Valencia, https://www.google.es/search?q=Arroyo +Zapatero, + L.: $+\%$ E2\% $80 \% 9 \mathrm{CLa}+$ violencia + de + g\%C3 $\%$ A9nero + en + la + pareja + en + el + Derecho + Penal + Espa\%C3\%B1ol\&ie $=u t f-8 \& o e=u t f-8 \& g w s$ rd=cr\&ei=L6AXV-7YE8LWUb6DhugI (página consultada el 20/04/16). El informe emitido por la mayoría del Consejo General del poder judicial en relación con el anteproyecto de Ley contra la violencia de género es señalado por el autor como un informe furioso, al considerar que toda la ley y especialmente las cláusulas punitivas y procesales se fundamentan en una discriminación de sexo. 
medida de discriminación positiva ${ }^{35}$ a favor de las mujeres, y se mostraban contrarios, por entender que se realizaba un tratamiento diferente y desfavorable en función del sexo, en este caso del masculino, en el ámbito del Derecho penal.

La posición del Consejo General era y sigue siendo compartida por algunos sectores del ámbito jurídico. Sin embargo, la cuestión hay que plantearla desde una perspectiva dogmático-jurídica diferente ${ }^{36}$ : hay que acudir al concepto de violencia de género. Si retomamos las definiciones examinadas en este texto puede comprobarse que la violencia de género —o contra las mujeres- es ejercida por un hombre contra una mujer, el sujeto activo necesariamente es un hombre y el sujeto pasivo es una mujer; y ello viene condicionado no tanto por el sexo si no por el género, esto es, por la atribución de funciones sociales establecidas por el sistema patriarcal a las personas de sexo masculino y femenino.

El que las mujeres sean el sujeto pasivo de la violencia de género y por tanto víctimas, no excluye la existencia de otras personas que también puedan ser víctimas, como niños, ancianos,... etc. Los hombres que quieren someter a las mujeres pueden ejercer varios tipos de violencia, incluido el maltrato de personas que se relacionan con ellas: hijos, padres, amigas, amantes, y un largo etcétera. El objetivo de tales maltratos es someterlas a una presión insoportable para que cedan a sus peticiones y, en definitiva, hacer efectiva su posición de poder.

El sistema patriarcal ha situado al sexo masculino en una posición de poder y al sexo femenino en una posición de subordinación en cada uno de los grupos de pertenencia; en cualquier tipo de organización social los hombres están en una posición de poder superior a las mujeres. Aunque exista algún caso concreto en que alguna mujer esté en posición superior, no deja de ser una excepción que,

35 Por el contrario el TC no considera que sea una acción positiva. Así, La STC 59/2008, de 14 de mayo, en la que desestima una cuestión de inconstitucionalidad (BOE de 4 de junio 2008), no entra a considerar la cuestión de si se trata de una acción positiva o de discriminación inversa sino que se trata de una aplicación de la igualdad real y efectiva propia del Estado Social y para ello es necesario establecer diferencias de trato. Para una ampliación de esta cuestión se puede consultar Sevilla Merino, J; García Campá, S.; y Ventura Franch, A. (2007). «La igualdad efectiva entre mujeres y hombres desde la teoría constitucional», en Revista del Ministerio de Trabajo e Inmigración, núm. 67, op. cit., pp. 63-82; y Sevilla Merino, J, y Ventura Franch, A. (2007). «Fundamento Constitucional de la Ley Orgánica 3/2007, de 22 de marzo, para la igualdad efectiva de mujeres y hombres. Especial referencia a la participación política». Revista del Ministerio de Trabajo e Inmigración, núm. extra 2, pp. 15-51.

36 Bodelón, E. (2012). Violencia de género y las respuestas de los sistemas penales. Op. cit., p. 17. La autora cuestiona si el derecho penal moderno entiende cuáles son las características de la violencia machista y su particularidad. Barrère, MA. (2008) «Iusfeminismo y derecho antidiscriminatorio: hacía la igualdad por la discriminación» en Mujeres, derechos y ciudadanía, Mestre, R. (coord.). Valencia. Tirant lo Blanc. 
en definitiva, confirma la regla; por tanto, se puede afirmar que los hombres, en el sistema sexo/género, ostentan el poder $^{37}$.

A partir de esta elaboración doctrinal, el origen de la violencia de género está en el sistema sexo/género, que determina y condiciona toda la estructura social y no sólo el ámbito de las relaciones afectivas; la violencia de género abarca a todas las violencias de las que se vale el patriarcado para mantener la posición de poder.

De acuerdo, pues, con el concepto de violencia de género defendido en este trabajo, los sujetos —activo y pasivo- son claramente identificables con cada uno de los sexos y, además, no pueden ser intercambiables: el varón tendrá una posición de poder y consecuentemente será el sujeto activo y la mujer tendrá una posición de subordinación y será el sujeto pasivo.

Esto no excluye que las mujeres también pueden ejercer violencia en general y por supuesto contra los varones, e indudablemente en tales casos deban ser castigadas por ello. Pero la violencia que pueden ejercer las mujeres nunca - $\mathrm{O}$ al menos mientras subsistan las posiciones de poder del sexo masculino y de subordinación del sexo femenino ${ }^{38}$ — podrá ser conceptualizada como violencia de género, dado que lo determinante para la catalogación de cualquier manifestación agresiva contra una persona como violencia de género no es el sexo de la persona que agrede, sino la pertenencia de ese sexo al grupo dominante. Es esto último lo que agrava de alguna manera el hecho de la agresión.

La violencia de género se puede dar en cualquier ámbito y la violencia que pueden ejercer las mujeres contra los hombres también, pero ello no debe conducirnos a la confusión de que toda violencia se pueda catalogar como violencia de género. La violencia de género presenta una gravedad diferente, aunque el resultado de la violencia sea el mismo; tal es el caso en que como consecuencia

37 AsúA, A. (2008). «El significado de la violencia sexual contra las mujeres y la reformulación de la tutela penal en este ámbito», en Género, violencia y derecho, op. cit., p. 131. La autora afirma que mantener la óptica de la desigualdad estructural de las asignaciones de género no supone desconocer la interacción de otros factores situacionales concretos que recorren las manifestaciones violentas, factores que explican la distinta incidencia de la violencia en función de otras formas de vulnerabilidad en la que se sitúan de forma diversa los distintos grupos de mujeres.

38 En cualquier caso, aunque se produjera una inversión en las relaciones de poder — cosa improbable y además no deseable, por cuanto la propuesta de la teoría feminista es acabar con las relaciones de dominación y no invertir el sistema de poder a favor de las mujeres-, necesariamente habría que volver a «reconceptualizar» la violencia de género, ya que algunos de los elementos que ahora forman parte del concepto de violencia de género no serían aplicables de forma automática si el sujeto activo de la violencia de género fueran las mujeres. 
de la violencia se produzca la muerte de una persona. La violencia que se ejerce desde una posición de poder tiene unas connotaciones más graves ${ }^{39}$.

La agravación de las penas en función de determinadas circunstancias no es ajena al derecho penal; así, se ha tenido en cuenta, a efectos de agravación de las penas, la pertenencia a grupos terroristas, tener relaciones de parentesco entre agresores y víctimas, o por razón de raza. Curiosamente, cuando se trata de agravar las penas para el caso de las agresiones por violencia de género es cuando afloran todos los temores y se acaba cuestionando la constitucionalidad de tales medidas.

Este debate se dio en nuestro país con la entrada en vigor de la Ley Integral Contra la Violencia de Género y la consecuente modificación del Código Penal. La oposición por parte de jueces y magistrados ${ }^{40}$ del Poder judicial se mostró en cuestiones de inconstitucionalidad planteadas al Tribunal Constitucional, que ha tenido ocasión de pronunciarse acerca de la constitucionalidad de la Ley en la STC 59/2008, de 14 de mayo.

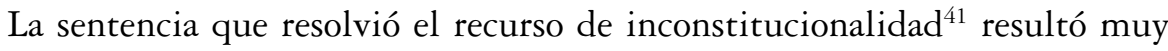
llamativa a una gran parte de la doctrina porque introduce la perspectiva de género para justificar la diferencia de trato desde la norma, en este caso penal. Sin embargo, desde nuestro punto de vista, la novedad se circunscribe a la cita-

39 Maqueda, L. (2007). « ¿Es la estrategia penal una solución a la violencia contra las mujeres?». InDret, Revista para el Análisis del Derecho, núm. 4, http://www.indret.com/pdf/475_ es.pdf, página consultada el 20/04/16.

40 Oposición, por lo demás, previsible, a tenor del debate producido en el seno del Consejo General del Poder Judicial en relación con el Informe emitido con ocasión del anteproyecto de Ley, donde ya se había visualizado la polarización de posturas.

${ }^{41}$ El recurso fue remitido por el Juzgado de lo penal núm. 4 de Murcia, al que se acompaña el Auto del mismo órgano jurisdiccional, de 29 de julio de 2005, por el que se plantea cuestión de inconstitucionalidad en relación con el art. 153.1 del Código Penal. La redacción del artículo 153.1 CP entonces vigente expresaba que «El que por cualquier medio o procedimiento causare a otro menoscabo psíquico o una lesión no definidos como delito en este Código, o golpeare o maltratare de obra a otro sin causarle lesión, cuando la ofendida sea o haya sido esposa, o mujer que esté o haya estado ligada a él por una análoga relación de afectividad aun sin convivencia, o persona especialmente vulnerable que conviva con el autor, será castigado con la pena de prisión de seis meses a un año o de trabajos en beneficios de la comunidad de treinta y uno a ochenta días y, en todo caso, privación del derecho a la tenencia y porte de armas de un año y un día a tres años, así como, cuando el Juez o Tribunal lo estime adecuado al interés del menor o incapaz, inhabilitación para el ejercicio de la patria potestad, tutela, curatela, guarda o acogimiento hasta cinco años». El auto se fundamenta en la posible infracción del art. $14 \mathrm{CE}$, señalando que el derecho a la igualdad que consagra se ve conculcado en razón de la discriminación por razón de sexo que dimana de la definición de los sujetos activo y pasivo en el precepto cuya constitucionalidad se cuestiona. 
ción expresa por parte del Tribunal de la perspectiva de género. A nuestro entender, era necesario que incluyera la perspectiva de género, por una parte, porque es la única explicación desde un criterio estrictamente científico de la existencia de una violencia estructural contra las mujeres vinculado al patriarcado; y, por otra, porque es desde ella que la violencia de género ha sido definida y codificada en la legislación española. La ley impugnada (LOIVG), en su artículo uno introduce la definición de la violencia de género como una consecuencia del sistema patriarcal, aunque la circunscribe al supuesto de las relaciones afectivas $^{42}$. Sin embargo, y a pesar de ello, el Tribunal no utiliza la perspectiva de género de manera automática para justificar la diferencia de trato, sino que vuelve a referirse a cada uno de los criterios que debe reunir la legitimidad constitucional de la diferencia de trato, a saber: 1) desigualdad en los supuestos de hecho; 2) finalidad de la medida; 3) adecuación de la medida a los fines; 4) proporcionalidad y razonabilidad de la misma; 5) con la finalidad de conseguir la igualdad.

Como ocurre con la aplicación del derecho antidiscriminatorio ${ }^{43}$, el Tribunal se considera en la necesidad de testar la medida concreta a fin de poder justificar su constitucionalidad. Y esto ocurre porque la presunción de constitucionalidad sigue siendo la de la igualdad formal: toda medida que rompe con la estricta igualdad formal hay que justificarla. Resulta harto difícil, desde el punto de vista jurídico, desvelar situaciones que sufren algunos individuos por su pertenencia a un grupo y cuyas discriminaciones tienen un origen grupal, en la medida que el derecho parte de la idea de sujetos individuales.

Ello explica el esfuerzo que el TC ha realizado en los fundamentos jurídicos de la sentencia para justificar que son las circunstancias y no los sujetos los que condicionan la agravación de la pena, y por ello comienza afirmando la existencia de dos contenidos diferenciados ${ }^{44}$ en el art. $14 \mathrm{CE}$ : el principio de igualdad y las prohibiciones de discriminación (Fj.5). El primer inciso incluye una cláusula general de igualdad de todos los españoles ante la ley, lo que comporta un derecho subjetivo de la ciudadanía a obtener un trato igual, que obliga y limita a los

42 Aranda, E. (2006), «Objeto y principios rectores de la ley integral», en ARANDA, E. (dir.), Estudios sobre la ley integral de la violencia de género, op. cit., p. 24. El autor afirma que la ley no se preocupa de todos los actos violentos de los hombres que conlleven un desvalor de la mujer.

43 Tal y como señala BARrère, M. A. en (2011). «Subordiscriminación y discriminación interseccional elementos para una teoría del derecho antidiscriminatorio». Anales de la Cátedra Francisco Suárez, núm. 45, pp. 15-42.

44 Doctrina establecida en la STC 128/87. Rodríguez PIÑEIRO, M. (1993). «Jurisprudencia del Tribunal Constitucional sobre discriminación laboral por razón de sexo». Revista Emakunde, Instituto Vasco de la Mujer, abril 1993, p. 34. 
poderes públicos a respetarlo, con la exigencia de que los supuestos de hecho iguales sean tratados de igual forma; y que para introducir diferencias entre ellos debe existir una suficiente justificación de tal diferencia, que aparezca al mismo tiempo como fundada y razonable de acuerdo con criterios y juicios de valor generalmente aceptados.

El Tribunal acepta que excepcionalmente el sexo discriminado puede ser un criterio de diferenciación jurídica $(\mathrm{Fj} 5)$, siguiendo la doctrina elaborada con anterioridad (STC 128/87, entre otras) y aplicando un canon más estricto en el control de constitucionalidad.

La justificación del TC se basa en que no es el sexo el que determina una diferencia de trato, sino el hecho de que ciertas «agresiones concretas que se producen en el seno de la pareja, o entre quienes lo fueron, al entender del legislador, como fundamento de su intervención penal, que las mismas se insertan en ciertos parámetros de desigualdad tan arraigados como generadores de graves consecuencias, con lo que aumenta la inseguridad, la intimidad y el menosprecio que sufre la víctima» (Fj11).

En definitiva, es la perspectiva de género la que justifica la diferencia de trato; pero, una vez aceptada y codificada (el art. 1 de la LOIVG), ya no era necesario reiterar la justificación del trato diferente en base a que el sexo del sujeto es o no el determinante y si la mujer podía también ser o no sujeto activo del delito de lesiones. La agravación de las penas precisamente tiene justificación en el género, que introduce una diferencia en los supuestos de hecho. No es, por tanto, la personalidad del sujeto activo lo que condiciona la pena, alejando la posibilidad de acercamiento al denominado derecho penal de autor, sino la posición de dominio que ostenta sobre la víctima.

Por ello, entendemos que la inclusión en un mismo tipo penal ${ }^{45}$ refleja la no integración de los conceptos de violencia de género y patriarcado en el tratamiento penal de las agresiones producto de este tipo de violencia. Consideramos que el legislador debía haber distinguido de manera clara la violencia de género de otros tipos de violencia y haber creado un tipo diferente, claramente vinculado al concepto de violencia de género, sobre el que no habría dudas acerca del sexo del sujeto activo y pasivo.

45 La modificación del Código Penal que se produce a partir de la entrada en vigor de la Ley Orgánica $1 / 2015$, de 30 de marzo, mantiene la misma tipificación en el los arts. 147 y 148; aunque incorpora el género como motivo de discriminación en las circunstancias agravantes del art. 22.4 del CP. Esta modificación introduce algunas novedades aunque no son motivo de análisis en este trabajo. 


\section{LA CONFUSIÓN CONCEPTUAL DE LA VIOLENCIA DE GÉNERO EN EL CONVENIO DE ESTAMBUL: LA CATEGORIZACIÓN Y LOS SUJETOS DE LA VIOLENCIA DOMÉSTICA}

\section{El Convenio del Consejo de Europa Sobre Prevención y Lucha Contra La} Violencia Contra La Mujer ${ }^{46}$ y La Violencia Doméstica, también denominado Convenio de Estambul, abierto a la firma en Estambul el 11 de mayo de 2011, entró en vigor en España el 1 de agosto de $2014^{47}$.

El Convenio nació en el seno de la Red parlamentaria por el derecho de las mujeres a vivir sin violencia del Consejo de Europa ${ }^{48}$. Ha sido denominado «estándar de oro» por ONU Mujeres, otorgándosele el Premio Política del Futuro $2014^{49}$, en reconocimiento al hecho de que en la actualidad es el instrumento internacional más amplio sobre la violencia contra las mujeres ${ }^{50}$, y con capacidad para influir en las legislaciones internas de los países firmantes así como para controlar los avances y los incumplimientos en la materia.

46 Llama la atención la utilización de «violencia contra la mujer» en el enunciado del Convenio y no «violencia contra las mujeres». El texto del Convenio, sin embargo, utiliza la palabra «mujeres» $\mathrm{y}$ «mujer» indistintamente; a veces hace referencia a «la mujer» y a veces a «las mujeres» [arts. 3, a); 4.1 y.2; 6, ..etc.]. Siguiendo a Celia Amorós, se puede afirmar que la utilización de la palabra «mujer», en singular, rememora cómo se configura la individualidad en la sociedad patriarcal. Las mujeres se desarrollan en el espacio privado, que aparece como el del ser social negado; no hay discernimiento de individuos ni sujetos. Se trata, pues, del espacio de las idénticas, lo que les niega la condición de individuo y las relega del espacio de los iguales. Amorós, C. (1987). «Espacio de los Iguales, espacio de las idénticas. Nota sobre poder y principio de individuación». Arbor, CXXVIII, pp. 113-127.

47 BOE de 6 de junio de 2014, núm. 137.

48 Consejo de Europa. Red Parlamentaria «Mujeres libres de violencia» (2013). Libre de miedo. Libre de violencia. Manual para parlamentarios, Estrasburgo, Consejo de Europa, (http://website-pace.net/documents/19879/730532/HandbookParliamentarians_SP.pdf/ cd0e31e8-968f-49b3-885b-4b83159249cd, página consultada el 20/04/16).

49 https://www.worldfuturecouncil.org/p/2014-ending-violence-against-women/. Página consultada el 21/03/16.

${ }^{50}$ Es, como se ha señalado, hasta la fecha, la regulación más extensa y más completa contra la violencia hacia las mujeres. Consta de un preámbulo, ochenta y un artículos y un anejo. El texto se organiza en XII capítulos. Su capítulo I está dedicado a «Objetivos, definiciones, igualdad y no discriminación, obligaciones generales»; el capítulo II, a «Políticas integradas y recogida de datos»; el III, a «Prevención»; el IV a «Protección y apoyo»; el V, a «Derecho material»; el VI, a «Investigación, procedimientos, derecho procesal y medidas de protección; el VII, a «Migración y asilo; el VIII, a «Cooperación internacional»; el IX, al «Mecanismo de seguimiento»; el X, a «Relación con otros instrumentos internacionales»; el XI, a «Enmiendas al Convenio; y el XII a «Cláusulas finales». 
Representa un gran avance en el ámbito internacional. Además, en algunos aspectos resulta especialmente interesante por las relaciones que establece, al vincular la eliminación de la violencia con la promoción de la igualdad. Así, en los objetivos del Convenio, entre otros, se señala su contribución a eliminar toda forma de discriminación contra la mujer y promover la igualdad real de mujeres y hombres, incluyendo su empoderamiento.

Que el Convenio establezca este objetivo significa que sitúa claramente el origen de la violencia de género en la desigualdad y, como consecuencia, en la discriminación de las mujeres. Sin embargo, esta clarividencia desaparece cuando en su artículo 3 define, a los efectos del Convenio, el concepto de violencia; los sujetos activos y pasivos de la misma; y la violencia doméstica, a la que le reconoce una entidad equiparable a la violencia de género o violencia contra la mujer.

De una lectura sosegada del Convenio se desprende una regulación de la violencia doméstica como diferenciada claramente de la violencia contra la mujer y desvinculada de la violencia de género, cuando, en realidad, como hemos visto, no es sino una de sus manifestaciones, bien cierto que muy extendida y también muy analizada.

Los redactores del Convenio no parecen entenderlo de esa manera y le dan a la violencia doméstica una entidad equivalente a la violencia de género; además, se incluye en su articulado que las víctimas de este tipo de violencia pueden ser tanto las mujeres como los hombres; no concreta, sin embargo, si las mujeres pueden ser, también, sujetos activos de la misma.

El preámbulo del Convenio hace referencia a la normativa del Consejo de Europa sobre la materia, a los principios básicos del Derecho humanitario internacional y a jurisprudencia del Tribunal Europeo de Derechos humanos en materia de violencia contra las mujeres. Todo ello sirve para fundamentar la creación de este Convenio, en el que se señala como finalidad una aspiración muy ambiciosa: crear una Europa libre de violencia contra la mujer.

Para ello, el Preámbulo incorpora unas declaraciones en las que sitúa, correctamente, el origen de la violencia. Así, en los «Reconociendo» Primero, Segundo y Tercero, textualmente ${ }^{51}$, se señala:

«Reconociendo que la realización de jure y de facto de la igualdad entre mujeres y hombres es un elemento clave de la prevención de la violencia contra la mujer;

Reconociendo que la violencia contra la mujer es una manifestación de desequilibrio histórico entre la mujer y el hombre que ha llevado a la dominación

51 BOE núm. 137, viernes, 6 de junio de 2014, Sec. I., p. 42946. 
y a la discriminación de la mujer por el hombre, privando así a la mujer de su plena emancipación;

Reconociendo que la naturaleza estructural de la violencia contra la mujer está basada en el género, y que la violencia contra la mujer es uno de los mecanismos sociales cruciales por los que se mantiene a las mujeres en una posición de subordinación con respecto a los hombres...»

Esa fundamentación y la finalidad de crear una Europa libre de violencia induce a pensar que el Convenio ha incorporado el concepto de violencia de género; en cambio, la lectura de los «Reconociendos» Seis y Siete produce una cierta confusión, ya que en ellos se realizan afirmaciones que desvirtúan los anteriores, a la vez que se apartan del concepto de violencia de género o violencia contra las mujeres ${ }^{52}$.

Dicha confusión se concreta después en el artículo 3, en el que se establecen las definiciones y los conceptos de violencia a los efectos del Convenio. Así, puede afirmarse que el «Reconociendo» Sexto anticipa la confusión conceptual entre violencia de género y violencia doméstica, cuando señala «que las mujeres y las niñas están más expuestas que los hombres a un riesgo elevado de violencia basada en el género». Y el «Reconociendo» Séptimo se refiere a la violencia doméstica señalando «que la violencia doméstica afecta a las mujeres de manera desproporcionada y que los hombres pueden ser también víctimas de violencia doméstica». Aquí el Convenio ya anuncia que la violencia doméstica es una categoría diferente a la violencia de género y, además, afirma que los hombres pueden ser también víctimas de la violencia doméstica.

Pero de acuerdo con el concepto de violencia de género y los sujetos de esta violencia que hemos analizado anteriormente —en la que, recordemos, se incluye como uno de sus tipos la violencia doméstica-, los hombres no están expuestos a ella; porque esta violencia, para que sea calificada como tal debe ser infligida por un hombre contra una mujer, dado que son ellos los que forman parte del grupo dominante y no las mujeres. Eso, como también se ha señalado anteriormente, no significa que se esté negando la posibilidad de que algunas mujeres puedan infligir violencia contra los hombres y que deban ser castigadas por ello; pero esta violencia no se puede conceptualizar como violencia de género. La inclusión de los hombres como posibles víctimas sólo puede entenderse en los casos en que un hombre sufra violencia como consecuencia de tener algún vín-

52 Esto es, la violencia que tiene su origen en la organización patriarcal de la sociedad y que se produce como consecuencia de la situación de desigualdad que tienen las mujeres respecto de los hombres (ONU). Declaración sobre la eliminación de la violencia contra la mujer. Adoptada por la Asamblea General de Naciones Unidas en 1993. 
culo con una mujer a la que su agresor pretende maltratar a través de las personas que con ella se relacionan; pero en ningún caso podría calificarse como tal el supuesto en que sea una mujer la que agrede a un hombre: sería violencia, pero no de género, cómo se ha señalado anteriormente.

Realmente, donde se focaliza y concreta la confusión conceptual entre violencia de género y doméstica así como en lo que respecta a los sujetos activos y pasivos, es en el Artículo. 3, «Definiciones (A los efectos del presente Convenio)». El artículo incluye unas definiciones de violencia conectadas con una falta de claridad en cuanto a los sujetos activos y pasivos (agresor y víctima) que pueden dar lugar a interpretaciones erróneas; en una primera lectura, descontextualizada de la teoría sobre la violencia de género, se podría llegar a la conclusión, errónea, de que los sujetos hombre y mujer pueden ser intercambiables.

El artículo tiene seis apartados ${ }^{53}$, en los que se define la violencia contra la mujer, la violencia doméstica, el género, la violencia contra la mujer por razones de género, la víctima y el término «mujer». De su simple enunciado ya casi podríamos afirmar que tanta exhaustividad denota una cierta incoherencia terminológica, a la vez que se echa en falta otro apartado, que definiera quien es el agresor; porque de esta manera se eliminaría la ambigüedad que refleja el convenio respecto al sujeto activo y pasivo de la violencia. En ese sentido, se señala en el apartado e) del art. 3 que la víctima es «toda persona física», lo que incluye también a los hombres, e induce a dudar respecto de si las mujeres podrían también ser agresoras.

Ante tanta profusión definitoria y con ánimo de aportar una cierta coherencia, podemos afirmar que, en realidad, el artículo 3 se refiere a dos tipos de violencia: la de género y la doméstica (apartados a y b); y que en los demás apar-

53 Artículo 3, Definiciones: «A los efectos del presente Convenio: a) Por «violencia contra la mujer» se deberá entender una violación de los derechos humanos y una forma de discriminación contra las mujeres, y se designarán todos los actos de violencia basados en el género que implican o pueden implicar para las mujeres daños o sufrimientos de naturaleza física, sexual, psicológica o económica, incluidas las amenazas de realizar dichos actos, la coacción o la privación arbitraria de libertad, en la vida pública o privada; b) Por «violencia doméstica» se entenderán todos los actos de violencia física, sexual, psicológica o económica que se producen en la familia o en el hogar o entre cónyuges o parejas de hecho antiguos o actuales, independientemente de que el autor del delito comparta o haya compartido el mismo domicilio que la víctima; c) Por «género» se entenderán los papeles, comportamientos, actividades y atribuciones socialmente construidos que una sociedad concreta considera propios de mujeres o de hombres; d) Por «violencia contra la mujer por razones de género» se entenderá toda violencia contra una mujer porque es una mujer o que afecte a las mujeres de manera desproporcionada; e) Por «víctima» se entenderá toda persona física que esté sometida a los comportamientos especificados en los apartados a y b; f) El término «mujer» incluye a las niñas menores de 18 años. 
tados define el género, la violencia contra las mujeres por razón de género, la víctima y el termino mujer.

En efecto, la «violencia contra la mujer» definida en el apartado a) del art. 3. está en consonancia con el concepto de violencia de género defendido en este trabajo, y con ella queda claro que el sujeto activo es un hombre y la víctima una mujer, con independencia que también puedan darse casos de víctimas hombres (apartado e), por el motivo de tener alguna relación con la mujer a la que se le infringe la violencia.

Sin embargo, la definición de la violencia doméstica del apartado b) del art. 3 se aparta considerablemente del concepto de violencia de género antes expuesto, y queda como una categoría diferenciada de ésta y no como uno de sus tipos, tal y cómo se señala claramente en la Declaración de sobre la Eliminación de la Violencia contra la Mujer (ONU), en la Resolución del Parlamento Europeo sobre Tolerancia Cero (1997) y en la teoría feminista.

En efecto, aunque las clasificaciones de la violencia contra las mujeres nunca pueden ser exhaustivas porque puede materializarse de muchas formas diferentes - tal y como señala el «Estudio a fondo sobre todas las formas de violencia contra la mujer» ${ }^{54}$ _, la literatura especializada en la materia señala, al menos, una tipología de la violencia, física, sexual, psicológica, económica, estructural, espiritual, política e institucional, violencia simbólica y violencia social. Estas diferentes formas de violencia contra las mujeres se pueden dar en distintos contextos: violencia doméstica, en conflictos armados, en la sociedad, en el ámbito laboral, en los medios de comunicación, violencia institucional y violencia en las tradiciones culturales o prácticas tradicionales nocivas. De acuerdo con las probabilidades de ocurrencia durante el ciclo vital de las mujeres, pueden darse en la etapa prenatal, infancia precoz, niñez, adolescencia, edad adulta y anciani$\mathrm{dad}^{55}$. Dentro de estas clasificaciones, la violencia doméstica aparece como una violencia contra las mujeres que se produce en el ámbito de la familia, en las relaciones de pareja y en las relaciones de noviazgo, y puede afectar también a los menores.

54 Naciones Unidas. Asamblea General (2006). Estudio a fondo sobre todas las formas de violencia contra la mujer. Informe del Secretario General, p.19, http://www.cepal.org/mujer/noticias/paginas/1/27401/informesecregeneral.pdf, (20/04/16). En este estudio, en el cuadro de definiciones de la violencia contra la mujer se cita expresamente la «Resolución de la Asamblea General sobre la eliminación de la violencia contra la mujer en el hogar»; y reconoce que «la violencia en el hogar puede incluir privaciones económicas y aislamiento, y ese tipo de comportamiento puede constituir un peligro inminente para la seguridad, la salud o el bienestar de la mujer».

55 Bosch, E., Ferrer, V. y Almazora, A. (2006). El laberinto patriarcal, op. cit, p. 57. 
Cuando parece que ya hay un consenso incluso doctrinal y en algunos casos normativo, de que la violencia que se genera en el ámbito doméstico es un tipo de violencia que tiene su origen en el sistema sexo/género, se vuelve a dar importancia a este tipo de violencia, dándole categoría jurídica diferenciada del concepto de violencia de género o violencia contra las mujeres.

En este sentido, parece pues que el Convenio de Estambul represente un retroceso con respecto a la caracterización de la violencia doméstica ${ }^{56}$. Y sin embargo, carece de sentido que el Convenio introduzca sus propias definiciones, porque en vez de clarificar puede contribuir a crear una mayor confusión, como es el caso en la violencia doméstica. Debería haberse optado por reproducir los estándares conceptuales sobre la violencia de género y establecer en su Preámbulo las justificaciones de los mismos, procurando eliminar contradicciones.

Por todo ello, con el ánimo de clarificar los conceptos de violencia que incluye el Convenio, consideramos conveniente que el Gobierno Español proponga una enmienda a su artículo 3, de acuerdo con el procedimiento establecido en el artículo 72 del mismo Convenio, en el que se defina el concepto de violencia de género y se identifiquen correctamente sus diferentes tipologías y los sujetos activos y pasivos de dicha violencia.

Title:

The Istanbul Convention and the subjects of gender violence. The questioned legal category of domestic violence.

\section{Summary:}

1. Introduction. 2. Relationship between patriarchy and gender violence: the concept of gender violence. 3. Men, active subjects of gender violence. 4 . The conceptual confusion of gender violence in the Istanbul Convention: categorization and subjects of domestic violence.

${ }^{56}$ En este sentido, Lousada Arochena, J. F. (2014). «El derecho fundamental a vivir sin violencia de género». Anales de la Cátedra Francisco Suarez, 48 (2014), pp. 31-48 (http://revistaseug. ugr.es/index.php/acfs/article/view/2779/2896, 20/04/16), en referencia al art. 3 del Convenio de Estambul afirma: «Pero, aunque en la definición de «violencia doméstica» se encuentra ausente el sexo mujer, y se encuentra asimismo ausente la referencia al género, la violencia doméstica es una violencia de género contra la mujer». 


\title{
Resumen:
}

El artículo tiene por objeto analizar el concepto de violencia de género incluido en el Convenio del Consejo de Europa sobre Prevención y lucha contra la violencia contra la mujer y la violencia doméstica, hecho en Estambul el 11 de mayo de 2011. La conceptualización de la violencia de género es un hecho de suma relevancia que puede contribuir de manera importante a su erradicación. Por ello, el artículo realiza un análisis de los elementos que la doctrina científica ha señalado como básicos para la definición de la violencia de género y los sujetos que la ejercen y la sufren. Para determinar qué es la violencia de género es necesario partir de los conceptos mediante los cuales se construye argumentativamente y jurídicamente la violencia producto del patriarcado como estructura social y el género como consecuencia de esa organización. A partir de estos criterios se analiza la conceptualización de la violencia que recoge el Convenio, observando algunas contradicciones que podrían introducir elementos de confusión al elevar a categoría jurídica la violencia doméstica y no determinar los sujetos activos y pasivos de la violencia de género. En base a ello, se propone una interpretación del concepto de violencia regulado en el artículo 3 del Convenio de acuerdo con los parámetros establecidos por la doctrina para la violencia de género, a la vez que se propone iniciar el trámite de presentación de enmiendas para su modificación, previsto en el mismo Convenio.

\begin{abstract}
:
The article aims to analyze the concept of gender violence included in the Council of Europe Convention on preventing and combating violence against women and domestic violence, made in Istanbul on 11 May 2011. The conceptualization of gender-based violence is a fact of great importance, which can contribute significantly to its eradication manner. Therefore, the article analyzes the elements of scientific doctrine has identified as basic to the definition of gender violence and subjects who exercise and suffer. To determine what is gender violence is needed start from the concepts by which it is built juridically and argumentatively the violence product of patriarchy as a social structure and gender as a consequence of that organization. From these criteria the conceptualization of violence that includes the Convention is analyzed, observing some contradictions that could introduce elements of confusion by raising a legal category of domestic violence and not to determine the active and passive subjects of gender violence. On this basis, an interpretation of the concept of violence as specified in Article 3 of the Convention, according to
\end{abstract}


the parameters established by the doctrine to gender violence, while it intends to begin the process of tabling amendments proposed its modification provided for in the Convention itself.

\section{Palabras Clave:}

Patriarcado, violencia, género, igualdad, derecho a una vida libre de violencia.

\section{Keywords:}

Patriarchy, violence, gender equality, the right to a life free of violence. 